\title{
SIZE AND SHAPE FACTOR EXTREMES OF SPHEROIDS
}

\author{
DANIEL HLUBinKA \\ Charles University in Prague, Faculty of Mathematics and Physics, Department of Probability and Statistics, \\ Sokolovská 83, 18675 Praha 8, Czech Republic \\ e-mail: daniel.hlubinka@mff.cuni.cz \\ (Accepted October 31, 2006)
}

\begin{abstract}
In the paper we consider random prolate (oblate) spheroids and their random profiles. The limiting distribution of the extremal characteristics of the spheroids is related to the limiting distribution of the corresponding extremal characteristics of the profiles. The difference between the analysis of the prolate and oblate spheroids is discussed. We propose the possible application of the theoretical results.
\end{abstract}

Keywords: maximum domain of attraction, shape factor of spheroids, size of spheroids, stereology of extremes.

\section{INTRODUCTION}

Microscopic particles (inclusions) in a material may cause a serious damage to that material. The study of the extremes of certain characteristics of such particles is important since material damage is related to the extremal rather than to the mean characteristics of the microstructure. The principal problem is typically that the particles are not observed directly. Only the profiles of the particles appearing on a random planar section of the material can be observed.

The basic model of the particles is random balls characterized by their size. The problem of estimating the size of random balls observed through their profiles has been well known for a long time as Wicksell's corpuscle problem (see Wicksell, 1925; 1926). Recently the problem of predicting extremal size based on profiles has been studied extensively; (see e.g., Drees and Reiss, 1992; Takahashi and Sibuya, 1996; 2002; Kötzer and Molchanov, 2006), where both the theoretical aspects and the applications to materials science are discussed.

In a series of papers, Hlubinka (2003a;b; 2006); Hlubinka and Kotz (2006), we have discussed the extremes of size or shape factor of oblate spheroids rather than balls. We present a new development of the topic in this paper. In particular we complete the study of the extremal characteristics of prolate spheroids; some new results are also proved for oblate spheroids. The analyses of the extremal characteristics of the two classes of spheroids are compared.

\section{PROLATE AND OBLATE SPHEROIDS}

Random microscopic particles of spheroidal shape in a volume of an opaque material are considered.
We shall further restrict our study either to prolate or to oblate spheroids (see e.g., Cruz-Orive (1976) for arguments why the restriction is used). Let us suppose that the particle arrangement is stationary and isotropic, which means that the underlying point process of their centers is stationary and their orientation is a uniformly distributed random variable. Recall that a prolate spheroid is a spheroid with one major semi-axis whose length is denoted by $X$ and two equal minor semi-axes with length $V$. An oblate spheroid is characterized by two equal major semiaxes (denoted by $X$ ) and one minor semi-axis (denoted by $V$, again). In the paper we use yet another set of characteristics of such spheroids. Define the shape factor of the spheroid by $S=X^{2} / V^{2}-1$. In CruzOrive (1976) it is proposed to use the characteristics $(X, S)$ for oblate spheroids, while the pair $(V, S)$ is used for prolate spheroids. However, we use the pair $(X, S)$ beside the usual characteristics $(V, S)$ for prolate spheroids later in the study when it is more appropriate.

\section{STEREOLOGY OF SPHEROIDS}

It is a usual complication in materials science that spheroids cannot be observed directly. It is, however, possible to observe in a planar section of the material, the profiles of spheroids. The random profiles of both oblate and prolate spheroids are ellipses. These ellipses are usually characterized by the lengths $Y$ and $W$ of their major and minor semi-axes respectively. The characteristics used further in this paper are an analogy to the characteristics of spheroids, namely the shape factor $T=Y^{2} / W^{2}-1$ and the size ( $Y$ for oblate and $W$ for prolate spheroids).

Remark 1. Note that for a prolate spheroid the length of the minor semi-axis is used as a size 
characteristic rather than the length of the major semiaxis, which seems to be the natural choice. Cruz-Orive (1976) explains why these characteristics are used in stereology. The main reason is that "the manipulation becomes much simpler" (Cruz-Orive, 1976). We will discuss the question of the size of prolate spheroid, in more detail later in the paper.

As used throughout the paper, the spheroid population consists either of prolate, or of oblate spheroids exclusively. Further, we consider the characteristics ( $V$ or $X$, and $S$ ) to be a random vector independent of the position and orientation of the particle. The bivariate distribution of the vector is considered to be absolutely continuous with the probability density function (density) denoted further by $g(v, s)$ and $g(x, s)$, respectively. The density of the profile characteristics $(W, T)$ or $(Y, T)$ is denoted by $f(w, t)$ and $f(y, t)$, respectively.

Let $M$ be the population mean size of the particles (half of the mean caliper diameter) and $x_{F}$ and $s_{F}$ the upper endpoint of the marginal distribution of the size $X$ and of the shape factor $S$ respectively. It can be determined e.g., in Hlubinka (2003a), that the density functions for the profile characteristics are

$$
\begin{aligned}
& f(w, t)=\frac{w}{2 M(1+t)^{2}} \int_{w}^{x_{F}} \int_{t}^{s_{F}} \frac{(1+s)^{3 / 2} g(v, s) d s d v}{\sqrt{s(s-t)} \sqrt{v^{2}-w^{2}}}, \\
& f(y, t)=\frac{y \sqrt{1+t}}{2 M} \int_{y}^{x_{F}} \int_{t}^{s_{F}} \frac{g(x, s) d s d x}{\sqrt{s(1+s)(s-t)} \sqrt{x^{2}-y^{2}}} .
\end{aligned}
$$

Remark 2. Note that the size $V$ of a prolate spheroid with a given shape factor $S=s$ cannot exceed the value $v_{F}(s)=x_{F} / \sqrt{1+s}$. Similarly the shape factor $S$ of a prolate spheroid with a given size $V=v$ cannot exceed the value $s_{F}(v)=x_{F}^{2} / v^{2}-1$ as follows from the definition. Hence the transformation $(\mathrm{P})$ should be rewritten as

$$
\begin{aligned}
f(w, t) & =\frac{w}{2 M(1+t)^{2}} \int_{t}^{s_{F}} \int_{w}^{v_{F}(s)} \frac{(1+s)^{3 / 2} g(v, s) d v d s}{\sqrt{s(s-t)} \sqrt{v^{2}-w^{2}}} \\
& =\frac{w}{2 M(1+t)^{2}} \int_{w}^{x_{F}} \int_{t}^{s_{F}(v)} \frac{(1+s)^{3 / 2} g(v, s) d s d v}{\sqrt{s(s-t)} \sqrt{v^{2}-w^{2}}} .
\end{aligned}
$$

This fact is not important when $x_{F}=\infty$ but it may otherwise cause a serious complication, as we shall see in the section "Application of the results".

\section{STABILITY OF MAXIMUM DOMAIN OF ATTRACTION}

Recall that if for a univariate distribution function $H$ there exist normalizing constants $a_{n}$ and $b_{n}$ such that, as $n \rightarrow \infty$ it holds that

$H^{n}\left(a_{n} u+b_{n}\right) \longrightarrow \begin{cases}\Lambda(u)=\exp \left(-\mathrm{e}^{-u}\right), & u \in \mathbb{R}, \\ \Phi_{\alpha}(u)=\exp \left(-u^{-\alpha}\right), & u \geq 0, \\ \Psi_{\alpha}(u)=\exp \left(-(-u)^{\alpha}\right), & u \leq 0,\end{cases}$

for some $\alpha>0$, then $H$ is said to belong to a maximum domain of attraction $(H \in \operatorname{MDA}(\cdot))$ of the limiting distribution function $\Lambda$ (Gumbel distribution), $\Phi$ (Fréchet distribution) or $\Psi$ (Weibull distribution), respectively. There are no other possible limiting distributions.

There are well known sufficient conditions for $H \in$ $\operatorname{MDA}(\cdot)$ if $H$ is an absolutely continuous distribution function with a density $h$ and an upper endpoint $z_{F}$. Consider the conditions

$$
\begin{array}{rlrl}
\lim _{z \nearrow z_{F}} \frac{h(z+u b(z))}{h(z)} & =\mathrm{e}^{-u}, & & u \in \mathbb{R}, \\
\lim _{z \rightarrow \infty} \frac{h(u z)}{h(z)} & =u^{-(\alpha+1)}, & u>0, z_{F}=+\infty, \\
\lim _{z \searrow 0} \frac{h\left(z_{F}-u z\right)}{h\left(z_{F}-z\right)} & =u^{\alpha-1}, & & u>0, z>0, z_{F}<+\infty,
\end{array}
$$

where $\alpha>0$. The detailed description of the auxiliary function $b(\cdot)$ in condition Eq. 2 can be found e.g., in Embrechts et al. (1997). In particular $b(\cdot)$ can be chosen such that it is differentiable for $z<z_{F}$, $\lim _{z \rightarrow z_{F}} b^{\prime}(s)=0$, and $\lim _{z \rightarrow \infty} b(z) / z=0$ if $z_{F}=\infty$, or $\lim _{z \rightarrow z_{F}} b(z) /\left(z_{F}-z s\right)=0$ if $z_{F}<\infty$. At most one of the conditions Eq. 2, Eq. 3 or Eq. 4 may hold for a density $h$.

The conditions are sufficient in the following sense:

$$
\begin{aligned}
& E q .2 \Rightarrow H \in \operatorname{MDA}(\Lambda), \\
& E q .3 \Rightarrow H \in \operatorname{MDA}\left(\Phi_{\alpha}\right),
\end{aligned}
$$

and

$$
E q .4 \Rightarrow H \in \operatorname{MDA}\left(\Psi_{\alpha}\right) \text {. }
$$

Remark 3. Throughout the paper we shall use the following notation for probability density functions:

- $g_{S}(s), f_{T}(t)$ are the marginal density functions of the shape factor of the spheroids and of the profiles, respectively; 
- $g .(s)$ and $f .(t)$ are the conditional densities of the spheroid's shape factor and of their profiles respectively, given an exact value of the size; and

- $g_{>} .(s)$ and $f_{>}(t)$ are the conditional densities of the shape factor of the oblate (prolate) spheroids and of their profiles respectively, given the size exceeds a given threshold. This case may be of particular interest for an application in the material sciences as the extremal shape factor is restricted to large particles only;

- We use the superscript $f^{o}(\cdot)$ for the density of oblate spheroids and $f^{p}(\cdot)$ for the density of prolate spheroids if it is not quite clear from the context which spheroid class is being considered.

We are now ready to prove a series of results concerning the stability of MDAs.

Theorem 4 (Shape factor of prolate spheroids I). Assume that the upper endpoint of the size distribution is $x_{F}=\infty$. Suppose further that the conditional density function $g_{v}(s)$ of the shape factor given the size $V=v$ satisfies one of the conditions Eq. 2, Eq. 3 or Eq. 4 uniformly in size. Then for the conditional density $f_{w}(t)$ of the profile shape factor given the size the same condition holds with a parameter $\beta$ replacing $\alpha$.

The value of $\beta$ is then $\beta=\alpha+1 / 2$ for both the Fréchet MDA and the Weibull MDA.

Remark 5. In fact, we will prove the "stability of the sufficient conditions" (2-4) up to a parameter rather than "the stability of the MDA". On the other hand it is possible but a bit more difficult to prove the stability of the equivalent conditions for the distribution functions; for these conditions see, e.g., Drees and Reiss (1992). Since the idea of such a proof is quite similar to the one presented in our proof, we prefer to use the simpler conditions (2-4).

Since the MDA can be estimated from the observed data (profiles) and since for the Weibull and Fréchet distributions the parameter can be also estimated based on the profiles, one can conclude that under the uniformity condition we also know the limiting distribution, and its parameter respectively, for the particle shape factor extremes.

Remark 6. The uniformity assumption of Theorem 4 (and of the similar theorems below) implies in particular that the parameter $\alpha$ of Eq. 3 and Eq. 4 is the same for all values of the size ( $x$ or $v$ respectively) and also that the auxiliary function $b(\cdot)$ of Eq. 2 is the same for all sizes. It also means that the upper endpoint $x_{F}$ does not depend on the size.
There are classes of bivariate distributions constructed by generalized Farlie-GumbelMorgenstern copulas for which the tail uniformity holds. The reader is referred to Hlubinka and Kotz (2006) for more details.

The tail uniformity condition in a similar sense may also be found for the extremal quantile regression, e.g., Chernozhukov (2005).

Remark 7. There is a natural question of what can happen without the tail uniformity assumption. In such a case it is possible that the spheroid's shape factor may belong to a different maximum domain of attractions for different sizes. As for the profile shape factor (when the profile size is fixed), the respective domain of attraction should reflect the heaviest tail of the spheroid's shape factor for which the spheroid size exceeds the observed profile size.

Proof of Theorem 4. We shall treat the three limiting cases separately. We use the fact that since $x_{F}=\infty$ the upper bound of the inner integral in (P1) is also infinite. Denote $\phi(v)=g_{V}(v) / \sqrt{v^{2}-w^{2}}$.

Fréchet limit: We need to study

$$
\begin{aligned}
& \lim _{t \rightarrow \infty} f_{w}(a t) / f_{w}(t)= \\
& =\lim _{t \rightarrow \infty} \frac{(1+t)^{2}}{(1+a t)^{2}} \frac{\int_{a t}^{\infty} \int_{w}^{\infty} \frac{(1+s)^{3 / 2} g(v, s) d v d s}{\sqrt{s(s-a t)} \sqrt{v^{2}-w^{2}}}}{\int_{t}^{\infty} \int_{w}^{\infty} \frac{(1+s)^{3 / 2} g(v, s) d v d s}{\sqrt{s(s-t)} \sqrt{v^{2}-w^{2}}}} \\
& =\lim _{t \rightarrow \infty} \frac{a(1+t)^{2}}{(1+a t)^{2}} \frac{\int_{w}^{\infty} \int_{t}^{\infty} \frac{(1+a s)^{3 / 2} g_{v}(a s) \phi(v) d s d v}{\sqrt{a s(a s-a t)}}}{\int_{w}^{\infty} \int_{t}^{\infty} \frac{(1+s)^{3 / 2} g_{v}(s) \phi(v) d s d v}{\sqrt{s(s-t)}}} \\
& =\lim _{t \rightarrow \infty} a^{-1 / 2} \frac{(1+t)^{2}}{\left(a^{-1}+t\right)^{2}} \frac{\int_{w}^{\infty} \int_{t}^{\infty} \frac{\left(a^{-1}+s\right)^{3 / 2} g_{v}(a s) \phi(v) d s d v}{\sqrt{s(s-t)}}}{\int_{w}^{\infty} \int_{t}^{\infty} \frac{(1+s)^{3 / 2} g_{v}(s) \phi(v) d s d v}{\sqrt{s(s-t)}}} \\
& =a^{-((\alpha+1 / 2)+1) .}
\end{aligned}
$$

The last equality follows from the fact that $\left(a^{-1}+\right.$ $t) /(1+t) \rightarrow 1$ as $t \rightarrow \infty$ and $g_{v}(a s) / g_{v}(s) \rightarrow a^{-(\alpha+1)}$ as $s \geq t \rightarrow \infty$ uniformly in $v$ and using Lemma 1.2.1 of de Haan (1970) for the limit of the ratio of integrals. 
Weibull limit: Using similar arguments as for Eq. 5 we obtain

$$
\begin{aligned}
& \lim _{t \searrow 0} f_{w}\left(s_{F}-a t\right) / f_{w}\left(s_{F}-t\right)= \\
& =\lim _{t \searrow 0} \frac{\left(1+s_{F}-t\right)^{2}}{\left(1+s_{F}-a t\right)^{2}} \frac{\int_{w}^{\infty} \int_{s_{F}-a t}^{s_{F}} \frac{(1+s)^{3 / 2} g(v, s) d s d v}{\sqrt{s} \sqrt{s-\left(s_{F}-a t\right)} \sqrt{v^{2}-w^{2}}}}{\int_{w}^{\infty} \int_{s_{F}-t}^{s_{F}} \frac{(1+s)^{3 / 2} g(v, s) d s d v}{\sqrt{s} \sqrt{s-\left(s_{F}-t\right)} \sqrt{v^{2}-w^{2}}}} \\
& =\lim _{t \searrow 0} \frac{a\left(1+s_{F}-t\right)^{2}}{\left(1+s_{F}-a t\right)^{2}} \frac{\int_{w}^{\infty} \int_{0}^{t} \frac{\left(1+s_{F}-a s\right)^{3 / 2} g_{v}\left(s_{F}-a s\right) \phi(v) d s d v}{\sqrt{s_{F}-a s} \sqrt{\left(s_{F}-a s\right)-\left(s_{F}-a t\right)}}}{\int_{w}^{\infty} \int_{0}^{t} \frac{\left(1+s_{F}-s\right)^{3 / 2} g_{v}\left(s_{F}-s\right) \phi(v) d s d v}{\sqrt{s_{F}-s} \sqrt{\left(s_{F}-s\right)-\left(s_{F}-t\right)}}} \\
& =\lim _{t \searrow 0} a^{1 / 2} \frac{\int_{w}^{\infty} \int_{0}^{t} \frac{\left(1+s_{F}-a s\right)^{3 / 2} g_{v}\left(s_{F}-a s\right) \phi(v) d s d v}{\sqrt{s_{F}-a s} \sqrt{t-s}}}{\int_{w}^{\infty} \int_{0}^{t} \frac{\left(1+s_{F}-s\right)^{3 / 2} g_{v}\left(s_{F}-s\right) \phi(v) d s d v}{\sqrt{s_{F}-s} \sqrt{t-s}}} \\
& =a^{\alpha-1 / 2}=a^{(\alpha+1 / 2)-1} .
\end{aligned}
$$

The second equality follows from the substitutions $s \leftrightarrow s_{F}-a s$ and $s \leftrightarrow s_{F}-s$ in the numerator and denominator, respectively. Lemma 1.2.1 of de Haan (1970) and the uniformity (in $v)$ of the limit $g_{v}\left(s_{F}-\right.$ as) $/ g_{v}\left(s_{F}-s\right) \rightarrow a^{\alpha-1}$ as $s \searrow 0$ complete the proof.

Gumbel limit: The situation is now complicated by the auxiliary function $b(\cdot)$. Recall (see $e . g$., Chapter 3 in Embrechts et al., 1997) that $b(s)$ can be chosen such that it is differentiable for $s<s_{F}$ and

$$
\lim _{s \rightarrow \infty} b^{\prime}(s)=0, \quad \lim _{s \rightarrow \infty} s^{-1} b(s)=0 \quad \text { if } s_{F}=\infty
$$

or

$\lim _{s \rightarrow s_{F}} b^{\prime}(s)=0, \quad \lim _{s \rightarrow s_{F}}\left(s_{F}-s\right)^{-1} b(s)=0 \quad$ if $s_{F}<\infty$.

Hence, using the second equality in Eq. 7 below the substitution $s \leftrightarrow s+a b(s)$ and the limit

$$
\lim _{t / s_{F}} \frac{1+t+a b(t)}{1+t}=1
$$

following from the properties of $b(\cdot)$, we obtain

$$
\begin{aligned}
& \lim _{t \nearrow s_{F}} f_{w}(t+a b(t)) / f_{w}(t)= \\
& =\lim _{t \nearrow s_{F}} \frac{(1+t)^{2}}{(1+t+a b(t))^{2}} \frac{\int_{w}^{\infty} \int_{t+a b(t)}^{s_{F}} \frac{(1+s)^{3 / 2} g_{v}(s) \phi(v) d s d v}{\sqrt{s} \sqrt{s-(t+a b(t))}}}{\int_{w}^{\infty} \int_{t}^{s_{F}} \frac{(1+s)^{3 / 2} g_{v}(s) \phi(v) d s d v}{\sqrt{s} \sqrt{s-t}}} \\
& =\lim _{t s_{F}} \frac{\int_{w}^{\infty} \int_{t}^{s_{F}} \frac{(1+s+a b(s))^{3 / 2}\left(1+a b^{\prime}(s)\right) g_{v}(s+a b(s)) \phi(v) d s d v}{\sqrt{s+a b(s)} \sqrt{(s+a b(s))-(t+a b(t))}}}{\int_{w}^{\infty} \int_{t}^{s_{F}} \frac{(1+s)^{3 / 2} g_{v}(s) \phi(v) d s d v}{\sqrt{s} \sqrt{s-t}}} \\
& =\exp \{-s\}
\end{aligned}
$$

by the Lemma 1.2.1 of de Haan (1970), again. In particular we need to check that

$$
\lim _{t \rightarrow s_{F}} \frac{(s+a b(s))-(t+a b(t))}{s-t}=1 \quad \text { where } \quad s>t .
$$

But this follows from the properties of $b(\cdot)$ : It holds that

$$
\frac{(s+a b(s))-(t+a b(t))}{s-t}=1+a b^{\prime}(\xi),
$$

for some $t \leq \xi \leq s$ and since $b^{\prime}(t) \rightarrow 0$ as $t \rightarrow s_{F}$, the proof is complete.

Remark 8. The case of oblate spheroids is simpler. There is no need to assume $x_{F}=\infty$ in analogy to Theorem 4; see Hlubinka (2003a). There is, however, a difference in the parameter change. In particular, the adjusted parameter is $\beta=\alpha$ for the Fréchet MDA and $\beta=\alpha+1 / 2$ for the Weibull MDA.

Very similar arguments can be used to prove the stability of MDA for the marginal density functions $g_{S}(s), f_{T}(t)$ and for the densities $g_{>\cdot(s)}$ and $f_{>\cdot}(t)$ of the shape factor conditioned by the size exceeding a given threshold.

Theorem 9 (Shape factor of prolate spheroids II). Suppose that the conditional density function $g_{v}(s)$ of the shape factor given the size $V=v$ satisfies one of the conditions Eq. 2, Eq. 3 or Eq. 4 uniformly in $v$ and for some parameter $\alpha>0$, respectively. Suppose moreover that the upper end point of the size distribution is $x_{F}=\infty$. Then

$i$. the marginal density $g_{S}(s)$ of the shape factor and the conditional densities $g_{>v}(s)$ of the shape factor given that the size exceeds a threshold, satisfy the same conditions (2-4) as $g_{v}(s)$ does. The respective parameter $\alpha$ of the limiting distribution is unchanged.

ii. the marginal density $f(t)$ of the profile shape factor and the conditional densities $f_{>w}(t)$ of the profile shape factor given the profile size exceeds a threshold satisfy the same conditions (2-4) as $g_{v}(s)$ does. The parameter $\beta$ of the limiting distribution is $\beta=\alpha+1 / 2$ for the Weibull and Fréchet MDAs.

Proof. The proof is an analogy to the proof of Theorem 4. We shall briefly show the main idea for the Fréchet limiting case.

The marginal density of the profile shape factor is

$$
f_{T}(t)=\frac{1}{2 M(1+t)^{2}} \int_{0}^{\infty} \int_{t}^{\infty} \frac{(1+s)^{3 / 2} v g(v, s) d s d v}{\sqrt{s} \sqrt{s-t}}
$$


The arguments of Eq. 5 can be repeated again and the proof for the marginal density functions can be concluded.

For the conditional density functions let us first note that

$$
\begin{aligned}
& g_{>v}(s)=\frac{\int_{v}^{\infty} g_{u}(s) g_{V}(u) d u}{\int_{v}^{\infty} g_{V}(u) d u}, \quad \text { and } \\
& f_{>w}(t)=\frac{\int_{w}^{\infty} f_{u}(t) f_{W}(u) d u}{\int_{w}^{\infty} f_{W}(u) d u}
\end{aligned}
$$

and that the denominators do not depend on the shape factor. Hence it is easy to find

$$
\lim _{s \rightarrow \infty} \frac{g_{>v}(a s)}{g_{>v}(s)}=\frac{\int_{v}^{\infty} g_{u}(a s) g_{V}(u) d u}{\int_{v}^{\infty} g_{u}(s) g_{V}(u) d u}=a^{-\alpha+1},
$$

again as a simple consequence of the uniformity assumption. The same argument may be used for the other two limiting cases.

For the last part of the proof it is sufficient to repeat the arguments in Eq. 5 line by line and to use the fact that $f_{>w}(t)=$

$$
\frac{1}{2 M(1+t)^{2}} \int_{t}^{\infty} \int_{w}^{\infty} \frac{(1+s)^{3 / 2} \sqrt{v^{2}-w^{2}} g(v, s) d v d s}{\sqrt{s} \sqrt{s-t}\left(\int_{w}^{\infty} f_{W}(u) d u\right)}
$$

Remark 10. There is an analogy to Theorem 9 for oblate spheroids again. The assumption $x_{F}=\infty$ may be omitted, as in Remark 8 . The parameter change is $\beta=\alpha$ for the Fréchet MDA and $\beta=\alpha+1 / 2$ for the Weibull MDA exactly as in Remark 8.

Remark 11 . The reason for the uniformity assumption of course can be seen from the proofs of Theorems 4 and 9. There is, however, also an intuitive reason for the uniformity assumption. Assume that we observe a profile with size $W=w$. Since it holds that $0 \leq w \leq V$, the observed spheroid may be of any size which is greater than or equal to the observed one. Hence the limiting behavior of the profile shape factor extreme is influenced by the limiting behavior of the shape factor extreme of any spheroid whose size is larger than the size of the observed profile. The uniformity is assumed in order to obtain just one MDA for the profile shape factor regardless of the actually observed size.

If the uniformity assumption is not valid then we may expect that there can be some dominating extreme value behavior for the shape factor extremes and that this dominating behavior is connected to some values of the size. But then it would be impossible to recover the non-dominating limiting behavior of the shape factor for the other (conditioning) values of the size.
Quite similar arguments hold for the size characteristics. There is no problem for the size of prolate spheroids if $x_{F}=\infty$ (hence the Weibull MDA is excluded).

Theorem 12 (Size of prolate spheroids I). Suppose that the upper endpoint $x_{F}=\infty$. Suppose that the conditional density function $g_{s}(v)$ of the size given the shape factor $S=s$ satisfies one of the conditions $E q .2$ or Eq. 3 uniformly in s and for some parameter $\alpha>1$ for Eq. 3. Then

$i$. the marginal density $g_{V}(v)$ of the size and the conditional densities $g_{>s}(v)$ of the size given that the shape factor exceeds the threshold s, satisfy the same of the conditions Eq. 2 or Eq. 3 as $g_{s}(v)$ does. The respective parameter $\alpha$ of the limiting distribution is unchanged.

ii. all the conditional densities $f_{t}(w)$ of the profile size given that the shape factor $T=t$, the marginal density $f_{W}(w)$ of the profile size and the conditional densities $f_{>t}(w)$ of the profile size given the profile shape factor exceeds the threshold t, satisfy the same of the conditions Eq. 2 or Eq. 3 as $g_{s}(v)$ does. The parameter $\beta$ of the limiting distribution is $\beta=\alpha-1$ for the Fréchet MDA.

Proof. The proof follows similar arguments as the proofs of Theorems 4 and 9, so it is sufficient to show the main steps only. We shall again distinguish the two limiting cases.

For the Fréchet MDA we can write

$$
\begin{aligned}
\lim _{w \rightarrow \infty} \frac{f_{t}(a w)}{f_{t}(w)} & =\lim _{t \rightarrow \infty} a \frac{\int_{t}^{s_{F}} \int_{a w}^{\infty} \frac{(1+s)^{3 / 2} g(v, s) d v d s}{\sqrt{s} \sqrt{s-t} \sqrt{v^{2}-a^{2} w^{2}}}}{\int_{t}^{s_{F}} \int_{w}^{\infty} \frac{(1+s)^{3 / 2} g(v, s) d v d s}{\sqrt{s} \sqrt{s-t} \sqrt{v^{2}-w^{2}}}} \\
& =a \lim _{w \rightarrow \infty} \frac{\int_{t}^{s_{F}} \int_{w}^{\infty} \frac{(1+s)^{3 / 2} g_{s}(a v) g_{S}(s) a d v d s}{\sqrt{s} \sqrt{s-t} \sqrt{a^{2} v^{2}-a^{2} w^{2}}}}{\int_{t}^{s_{F}} \int_{w}^{\infty} \frac{(1+s)^{3 / 2} g_{s}(v) g_{S}(s) d s d v}{\sqrt{s} \sqrt{s-t} \sqrt{v^{2}-w^{2}}}} \\
& =a \lim _{w \rightarrow \infty} \frac{\int_{t}^{s_{F}} \frac{(1+s)^{3 / 2} g_{S}(s)}{\sqrt{s} \sqrt{s-t}} \int_{w}^{\infty} \frac{g_{s}(a v)}{\sqrt{v^{2}-w^{2}}} d v d s}{\int_{t}^{s_{F}} \frac{(1+s)^{3 / 2} g_{S}(s)}{\sqrt{s} \sqrt{s-t}} \int_{w}^{\infty} \frac{g_{s}(v)}{\sqrt{v^{2}-w^{2}}} d v d s} \\
& =a^{-((\alpha-1)+1) .}
\end{aligned}
$$

as follows from the assumptions of the theorem. 
We can continue with the Gumbel MDA. Now due to the properties of $b(\cdot)$, we obtain

$$
\begin{aligned}
& \lim _{w \rightarrow \infty} f_{t}(w+a b(w)) / f_{t}(w)= \\
& =\lim _{w \rightarrow \infty} \frac{w+a b(w)}{w} \frac{\int_{t}^{s_{F}} \int_{w+a b(w)}^{\infty} \frac{(1+s)^{3 / 2} g(v, s) d v d s}{\sqrt{s} \sqrt{s-t} \sqrt{v^{2}-(w+a b(w))^{2}}}}{\int_{t}^{s_{F}} \int_{w}^{\infty} \frac{(1+s)^{3 / 2} g(v, s) d s d v}{\sqrt{s} \sqrt{s-t} \sqrt{v^{2}-w^{2}}}} \\
& =\lim _{t \rightarrow s_{F}} \frac{\int_{t}^{s_{F}} \int_{w}^{\infty} \frac{(1+s)^{3 / 2}\left(1+a b^{\prime}(s)\right) g_{s}(v+a b(v)) g_{S}(s) d v d s}{\sqrt{s} \sqrt{s-t} \sqrt{(v+a b(v))^{2}-(w+a b(w))^{2}}}}{\int_{t}^{s_{F}} \int_{w}^{\infty} \frac{(1+s)^{3 / 2} g_{s}(v) g_{S}(s) d v d s}{\sqrt{s} \sqrt{s-t} \sqrt{v^{2}-w^{2}}}} \\
& =\exp \{-s\}
\end{aligned}
$$

by the Lemma 1.2.1 of de Haan (1970), again. In particular we need to check that

$$
\lim _{w \rightarrow \infty} \frac{(v+a b(v))^{2}-(w+a b(w))^{2}}{v^{2}-w^{2}}=1
$$

when $v>w \rightarrow \infty$. But this follows from the properties of $b(\cdot)$. It holds that

$$
\begin{aligned}
& \frac{(v+a b(v))^{2}-(w+a b(w))^{2}}{v^{2}-w^{2}}= \\
& =1+2 a \frac{v b(v)-w b(w)}{(v-w)(v+w)} \\
& \quad+a^{2} \frac{(b(v)-b(w))(b(v)+b(w))}{(v-w)(v+w)} \\
& \rightarrow 1
\end{aligned}
$$

as $v \geq w \rightarrow \infty$ and since there exists $\xi \in(w, v)$ such that

$$
\frac{b(v)-b(w)}{v-w}=b^{\prime}(\xi) \rightarrow 0, \quad \text { as } \quad w \rightarrow \infty
$$

and also $b(w) / w \rightarrow 0$, as $w \rightarrow \infty$.

The proof for the conditional density $f_{>t}(w)$ is easy and follows from the fact that

$$
\begin{aligned}
& f_{>t}(w)= \\
& =\frac{w}{2 M} \int_{w}^{x_{F}} \int_{t}^{s_{F}} \frac{g(v, s)}{\sqrt{v^{2}-w^{2}}} \psi(s, t) d s d x \frac{1}{1-F_{T}(t)},
\end{aligned}
$$

where the term

$$
\psi(s, t)=\frac{1}{2 \sqrt{s}}\left[\frac{2 \sqrt{(s-t)(1+s)}}{1+t}+\log \frac{1+\sqrt{\frac{s-t}{1+s}}}{1-\sqrt{\frac{s-t}{1+s}}}\right]
$$

does not depend on $w$. The rest of the proof is the same as the proof of Theorem 9 for the Fréchet MDA. Since the arguments for the Gumbel MDA are similar, they can be omitted and the proof is complete.
Remark 13 (Size of oblate spheroids). There is an analogy to Theorem 12 for oblate spheroids again. For the marginal and conditional densities $g_{S}(s), f_{Y}(y)$ and $f_{t}(y)$ it is Hlubinka (2003b, Theorem 2 in). The same result can be proved for the conditional densities $g_{>s}(x)$ and $f_{>t}(y)$ using similar arguments as in the proof of Theorem 12. Recall that the parameter change is $\beta=\alpha-1$ for the Fréchet MDA (compare with the prolate case) and $\beta=\alpha+1 / 2$ for the Weibull MDA.

\section{PROLATE SPHEROIDS WITH BOUNDED SIZE}

We turn our attention to prolate spheroids with the size bounded from above. The situation is now much more difficult than the unbounded case. We summarize the essential differences in a remark.

Remark 14. The pairs $(X, V)$ and $(X, S)$ are in a oneto-one correspondence given by

$$
(V \sqrt{1+S}, S)=(X, S)=\left(X, X^{2} / V^{2}-1\right),
$$

and so are $(W, Y)$ and $(T, Y)$. In particular, having a spheroid with a given shape factor $S=s$ there is an upper bound for the minor semi-axis, namely $V \leq x_{F} / \sqrt{1+s}$. In this respect, there is no difference between oblate and prolate spheroids. But, and this is the main difference, the value of $V$ is also bounded from below for prolate spheroids by the value of the profile size $W$. It follows that observing a profile of a prolate spheroid with (profile) size $W=w$ the shape factor of the spheroid is limited from above by $S \leq x_{F}^{2} / w^{2}-1$. There is no similar bound for oblate spheroids. On the other hand, observing the profile of a prolate spheroid with (profile) shape factor $T=t$ it is clear that the size of the spheroid is limited from above by $V \leq x_{F} / \sqrt{1+t}$. Again there is no similar bound for oblate spheroids.

We shall see later that the fact $V \leq x_{F} / \sqrt{1+t}$ causes particular technical problems for the method of proof we have applied in section "Stability of maximum domain of attraction" when we were studying the profile size (or shape factor) given the profile shape factor (or size, respectively). There is, however, no evidence that "MDA stability" does not hold in such a case and in particular we suspect that, possibly under additional conditions, the analogies to Theorems 4, 9 and 12 may be proved using a different method of proof. 


\section{SIZE}

Let us now consider the length of the major semiaxis $X$ and the shape factor $S$ to be the characteristics of a prolate spheroid with joint density $g^{*}(x, s)$. The joint density of the analogous characteristics of the profiles then becomes (we use the superscript ${ }^{*}$ to emphasize that $x$ and $y$ are used as the size)

$$
\begin{aligned}
& f^{*}(y, t)=\frac{y}{2 M(1+t)^{5 / 2}} \times \\
& \int_{t}^{s_{F}} \int_{y}^{x_{F}} \sqrt{\frac{1+s}{1+t}} \frac{1_{\left[\sqrt{1+s} \leq \frac{x_{F}}{y}\right.} \sqrt{1+t]}(1+s)^{2} g^{*}(x, s) d x d s}{\sqrt{s(s-t)} \sqrt{(1+t) x^{2}-(1+s) y^{2}}},
\end{aligned}
$$

for $0 \leq t \leq s_{F}$, and 0 otherwise. We have denoted by $1_{[A]}$ the indicator function of a set $A$.

The main problem lies in the fact that for a given prolate spheroid the upper bound of the profile size $Y$ depends on the unknown shape factor $S$. In particular assuming a spheroid whose shape factor is $S=s$ then

$$
Y \leq x_{F} \sqrt{\frac{1+T}{1+s}}
$$

must hold. This fact does not hold for oblate spheroids!

The upper bound of the profile size $Y$ given the shape factor $T=t$ is $x_{F}$, but this value can be attained if and only if $T=S=t$. Since the lower limit of the inner integral in Eq. 12 depends on the integration variable $s$ of the outer integral, it seems to us that we cannot employ the approach used for oblate spheroids for the analysis of the tail behavior of profile size $Y$ given the profile shape factor $T=t$. In particular we don't know a direct way to find the relation between the tail behavior of the profile size $Y$ given the profile shape is $T=t$ and the tail behavior of the size $X$ given the shape is $S=s$.

Nevertheless we can "remove" the condition $T=t$ and use the marginal distribution of the profile size $Y$. This restriction means that we cannot relate the distribution of the size to the shape factor. On the other hand we are still able to prove the relation between the tail behavior of the profile size $Y$ and the spheroid size $X$, respectively. The marginal density function of the profile size $Y$ is

$$
\begin{aligned}
f_{Y}^{*}(y) & =\int_{0}^{s_{F}} f^{*}(y, t) d t \\
& =\frac{1}{3 y^{3} M} \int_{0}^{s_{F}} \frac{g_{S}(s)}{\sqrt{s(1+s)}} \int_{y}^{x_{F}} g_{s}(x) x \zeta_{y}(x) d x d s
\end{aligned}
$$

where

$$
\zeta_{y}(x)=2\left(x^{2}+y^{2}\right) \mathbb{E}_{E}\left[1-y^{2} / x^{2}\right]-y^{2} \mathbb{E}_{K}\left[1-y^{2} / x^{2}\right]
$$

and $\mathbb{E}_{E}, \mathbb{E}_{K}$ are the elliptic $\mathrm{E}$ and elliptic $\mathrm{K}$ functions, respectively.

Theorem 15 (Size of prolate spheroids II). Assume the upper endpoint $0<x_{F}<\infty$. Suppose that the conditional density function $g_{s}^{*}(x)$ of the size given the shape factor $S=s$ satisfies for some parameter $\alpha>0$ the condition Eq. 4 uniformly in s. Then

$i$. the marginal density $g_{X}^{*}(x)$ of the size also satisfies the condition Eq. 4. The parameter $\alpha$ of the limiting distribution is unchanged.

ii. the marginal density $f_{Y}^{*}(y)$ of the profile size also satisfies the conditions Eq. 4 with parameter $\beta$. The parameter $\beta$ of the limiting distribution is $\beta=\alpha+1$.

Proof. We show only the principal argument for (ii) since the idea of the proof is essentialy the same as for the other theorems.

We need to study $f_{Y}^{*}\left(x_{F}-a z\right) / f_{Y}^{*}\left(x_{F}-z\right) \approx$

$$
\begin{aligned}
& \frac{\left(x_{F}-z\right)^{3}}{\left(x_{F}-a z\right)^{3}} \frac{\int_{0}^{s_{F}} \psi(s) \int_{0}^{z} a g_{s}^{*}\left(x_{F}-a x\right) \xi_{a z}(a x) d x d s}{\int_{0}^{s_{F}} \psi(s) \int_{0}^{z} g_{s}^{*}\left(x_{F}-x\right) \xi_{z}(x) d x d s} \\
& \rightarrow a a^{\alpha-1}=a^{\alpha}, \text { as } z \searrow 0,
\end{aligned}
$$

where " $A(z) \approx B(z)$ " is used in the sense $\mid A(z) / B(z)-$ $1 \mid \rightarrow 0$, as $z \rightarrow 0$,

$$
\xi_{z}(x)=\left(2\left(x_{F}-x\right)^{2}-\left(x_{F}-z\right)^{2}\right)
$$

and $\psi(s)=g_{S}^{*}(s)[s(1+s)]^{-1 / 2}$ does not depend on $z$.

Remark 16. Comparing Theorem 15 and Remark 13 we can see that the parameter $\beta$ is different for oblate and prolate spheroids. In particular the tail of the profile size is lighter in the prolate case. The reason is in the fact that there are two major semiaxes in the oblate case and hence the profile size can attain its maximum $x_{F}$ regardless of the orientation of the sectioning plane respective to the orientation of the spheroid. On the other hand, in the prolate case the size $Y$ can reach the upper endpoint $x_{F}$ if and only if the sectioning plane is parallel to the major semiaxis of the spheroid. 


\section{SHAPE FACTOR}

The situation of the shape factor is quite similar. Hence we have to restrict our study to the MDA of the marginal distribution of the shape factor. For the conditional distribution of the shape factor given the size we encounter similar difficulties as for the size.

We shall use the length of the major semi-axis $X$ as the more natural choice of the size characteristic of the spheroid. The marginal distribution of the profile shape factor $T$ is obtained by integrating of $f^{*}(y, t)$, see Eq. 12, over $y$. Hence

$f_{T}^{*}(t)=\frac{1}{2 M(1+t)^{2}} \int_{t}^{s_{F}} \frac{1+s}{\sqrt{s(s-t)}} \int_{0}^{x_{F}} x g^{*}(x, s) d x d s$,

where $g^{*}(x, s)$ is the joint density of the random vector $(X, S)$. We can see that the limits of the inner integral do not depend on the unknown value of $S$. Now we can prove the following theorem.

Theorem 17 (Shape factor of prolate spheroids III). Assume that the upper endpoint of the size distribution satisfies $x_{F}<\infty$. Suppose further that the conditional density function $g_{x}^{*}(s)$ of the shape factor given the size satisfies one of the conditions Eq. 2, Eq. 3 or Eq. 4 uniformly in size. Then, for the marginal density $f_{T}^{*}(t)$ of the profile shape factor, the same conditions hold with a respective parameter $\beta$.

The parameter satisfies $\beta=\alpha+1$ for the Fréchet MDA and $\beta=\alpha+1 / 2$ for the Weibull MDA.

Remark 18. Comparing Theorems 4 and 17 we can see that only the result for the Fréchet MDA is different. In particular, the tail of the profile shape factor is lighter than the tail of the spheroid shape factor. The reason is probably in the fact that having an unbounded shape factor and bounded size the shape factor is extremal when $V$ is negligible. Note that a large profile shape factor is observed if the sectioning plane is almost parallel to the major semi-axis. But such an event is quite rare since $V$ is very small.

Proof. We show only the essential part, as the proof is quite similar to the proof of Theorem 4. For the Fréchet MDA we get

$$
\begin{aligned}
& \lim _{t \rightarrow \infty} f_{T}^{*}(a t) / f_{T}^{*}(t)= \\
& =\lim _{t \rightarrow \infty} \frac{(1+t)^{2}}{(1+a t)^{2}} \frac{\int_{0}^{x_{F}} x g_{X}^{*}(x) \int_{a t}^{\infty} \frac{(1+s) g_{X}^{*}(s) d s}{\sqrt{s} \sqrt{s-a t}} d x}{\int_{0}^{x_{F}} x g_{X}^{*}(x) \int_{t}^{\infty} \frac{(1+s) g_{x}^{*}(s) d s}{\sqrt{s} \sqrt{s-t}} d x} \\
& =\lim _{t \rightarrow \infty} \frac{(1+t)^{2}}{(1+a t)^{2}} \frac{\int_{0}^{x_{F}} x g_{X}^{*}(x) \int_{t}^{\infty} \frac{(1+a s) g_{x}^{*}(a s) a d s}{\sqrt{a s} \sqrt{a s-a t}} d x}{\int_{0}^{x_{F}} x g_{X}^{*}(x) \int_{t}^{\infty} \frac{\left(1+s g_{X}^{*}(s) d s\right.}{\sqrt{s} \sqrt{s-t}} d x} \\
& =a^{-(\alpha+2)}
\end{aligned}
$$

For the Weibull MDA it holds that

$$
\begin{aligned}
& \lim _{t \backslash 0} f_{T}^{*}\left(s_{F}-a t\right) / f_{T}^{*}\left(s_{F}-t\right)= \\
& =\lim _{t \searrow 0} \frac{\left(1+s_{F}-t\right)^{2}}{\left(1+s_{F}-a t\right)^{2}} \frac{\int_{0}^{x_{F}} \int_{s_{F}-a t}^{s_{F}} \frac{x g_{X}^{*}(x)(1+s) g_{x}^{*}(s) d s d x}{\sqrt{s} \sqrt{s-\left(s_{F}-a t\right)}}}{\int_{0}^{x_{F}} \int_{s_{F}-t}^{s_{F}} \frac{x g_{X}^{*}(x)(1+s) g_{x}^{*}(s) d s d x}{\sqrt{s} \sqrt{s-\left(s_{F}-t\right)}}} \\
& =\lim _{t \searrow 0} \frac{\left(1+s_{F}-t\right)^{2}}{\left(1+s_{F}-a t\right)^{2}} \frac{\int_{0}^{x_{F}} \int_{0}^{t} \frac{x g_{X}^{*}(x)\left(1+s_{F}-a s\right)_{X}^{*}\left(s_{F}-a s\right) a d s d x}{\sqrt{s_{F}-a s} \sqrt{\left(s_{F}-a s\right)-\left(s_{F}-a t\right)}}}{\int_{0}^{x_{F}} \int_{0}^{t} \frac{x g_{X}^{*}(x)\left(1+s_{F}-s\right) g_{x}^{*}\left(s_{F}-s\right) d s d x}{\sqrt{s_{F}-s} \sqrt{\left(s_{F}-s\right)-\left(s_{F}-t\right)}}} \\
& =a^{\alpha-1 / 2}=a^{(\alpha+1 / 2)-1} .
\end{aligned}
$$

Finally for the Gumbel case it holds that

$$
\begin{aligned}
& \lim _{t / s_{F}} f_{T}^{*}(t+a b(t)) / f_{T}^{*}(t)= \\
& =\lim _{t / s_{F}} \frac{(1+t)^{2}}{(1+t+a b(t))^{2}} \frac{\int_{0}^{x_{F}} \int_{t+a b(t)}^{s_{F}} \frac{x g_{X}^{*}(x)(1+s) g_{X}^{*}(s) d s d x}{\sqrt{s} \sqrt{s-(t+a b(t))}}}{\int_{0}^{x_{F}} \int_{t}^{s_{F}} \frac{x g_{X}^{*}(x)(1+s) g_{X}^{*}(s) d s d x}{\sqrt{s} \sqrt{s-t}}} \\
& =\lim _{t / s_{F}} \frac{\int_{0}^{x_{F}} \int_{t}^{s_{F}} \frac{x g_{X}^{*}(x)(1+s+a b(s))\left(1+a b^{\prime}(s)\right) g_{X}^{*}(s+a b(s)) d s d x}{\sqrt{s+a b(s)} \sqrt{(s+a b(s))-(t+a b(t))}}}{\int_{0}^{x_{F}} \int_{t}^{s_{F}} \frac{x g_{X}^{*}(x)(1+s) g_{X}^{*}(s) d s d x}{\sqrt{s} \sqrt{s-t}}} \\
& =\exp \{-s\} .
\end{aligned}
$$

The proof is complete.

\section{APPLICATION OF THE RESULTS}

In this section we discuss the possible application of the above theorems.

We are interested in the distribution of the extreme spheroid characteristics. Let us denote by $C$ the spheroid characteristics of interest and by $C_{n: n}$ the sample maximum. The corresponding profile characteristics are denoted by $D$ and the sample maximum is $D_{m: m}$. What can be said about $\mathrm{P}\left[C_{n: n}<x\right]$ for large $n$, based on the profile sample and using the results of the above theorems?

First of all, the extreme value index $\kappa$ for the profile characteristics $D$ can be estimated from the observed profiles. Recall that the sign of the extreme value index corresponds to the maximum domain of attraction. In particular for the Weibull MDA it holds that $\kappa<0$, $\kappa>0$ for the Fréchet MDA and $\kappa=0$ for the Gumbel MDA. The estimate $\widehat{\beta}$ of the respective parameter of the Fréchet or Weibull limiting distribution can also be based on the observed profiles. There are classical 
methods for these estimates; see e.g., Coles (2001) or Beirlant et al. (2004) for recent references. Then, under the uniformity conditions of the theorems, we can estimate the maximum domain of attraction for $C$, and its respective parameter is estimated by a simple transformation of $\widehat{\beta}$ as proved in the Theorems above.

Assume that the characteristic $C$ (and also $D$ ) belongs to the MDA $\Upsilon$, where $\Upsilon$ is one of the three possible limiting distributions. Hence, there exist normalizing constants $\left(a_{n}, b_{n}\right)$ such that

$$
\mathrm{P}\left[C_{n: n}<a_{n} x+b_{n}\right] \doteq \Upsilon(x) .
$$

Recall, that normalizing constants form a sequence of pairs $\left(a_{n}, b_{n}\right)$ such that Eq. 1 holds. To determine the normalizing constants, it is usually necessary to analyze the tail behavior of the distribution at hand.

We cannot proceed directly, as follows from the fact that we need to estimate the normalizing constants for the spheroid characteristics $C$ but we don't observe the tail behavior of $C$. We must base our estimates on the normalizing constants estimated from the tail behavior of the profile characteristics $D$.

The normalizing constants for the observed profile characteristics $D$ can be estimated in many ways. The two most common include the maximum likelihood estimator based on the $k$ largest observations and the estimators based on the $k$ blocks maxima; see, e.g., Coles (2001) for more details.

If $\left(\widehat{a}_{n}^{p}, \widehat{b}_{n}^{p}\right)$ are the estimated normalizing constants for the observed profile characteristics $D$, and $\Upsilon(\cdot)$ is the limiting distribution function then we need to calculate the normalizing constants $\left(\widehat{a}_{n}^{s}, \widehat{b}_{n}^{s}\right)$ for the spheroid characteristics $C$. This calculation must be based on some relation between $\left(\widehat{a}_{n}^{p}, \widehat{b}_{n}^{p}\right)$ and $\left(\widehat{a}_{n}^{s}, \widehat{b}_{n}^{s}\right)$. The tail of the corresponding distribution function provides us with information on the normalizing constants. In this connection a version of the following lemma is often useful. We quote the case of the Gumbel MDA.

Lemma 19 (Normalizing constants for the Gumbel MDA). Suppose that a distribution function $K \in$ $\operatorname{MDA}(\Lambda)$ has an infinite upper end point. If there exist constants $\alpha>0, \beta, \gamma>0, \delta>0$ such that

$$
\lim _{v \rightarrow \infty} \frac{1-K(v)}{\alpha v^{\beta} \mathrm{e}^{-\gamma v^{\delta}}}=1,
$$

then the normalizing constants can be chosen as

$$
\begin{aligned}
& a_{n}=\left(\frac{\log n}{\gamma}\right)^{1 / \delta-1} \frac{1}{\gamma \delta}, \\
& b_{n}=\left(\frac{\log n}{\gamma}\right)^{1 / \delta}+\frac{\frac{\beta}{\delta}(\log \log n-\log \gamma)+\log \alpha}{\left(\frac{\log n}{\gamma}\right)^{1-1 / \delta} \gamma \delta} .
\end{aligned}
$$

This lemma and its proof can be found in Takahashi (1987).

Similar results are also known for the other two MDA; see e.g., Embrechts et al. (1997) for a possible choice of the normalizing constants based on the quantile function. Lemma 19 is generalized by Lemma 3.8 in Hlubinka and Kotz (2006) which is of particular interest if the generalized Farlie-GumbelMorgenstern bivariate distribution is used for the spheroid's characteristics.

The procedure for the case of the Gumbel MDA can be the following. For some parametric model describing the asymptotic tail behavior of the spheroid characteristics we shall derive the formula for the normalizing constants $\left(a_{n}^{s}, b_{n}^{s}\right)$ using Lemma 19, where $n$ is considered to be a variable rather than a constant. The same Lemma 19 applied to the appropriate density function based on transformation $(\mathrm{O})$ or $(\mathrm{P})$, respectively, can be used to derive the formula for the normalizing constants $\left(a_{m}^{p}, b_{m}^{p}\right)$ for the profile characteristics. Comparison of these two pairs of normalizing constants will suggest how to adjust the estimated normalizing constants of the profile characteristics to the estimate of the normalizing constants of the spheroid characteristics. For a more detailed discussion of the recalculation of the normalizing constants, see Hlubinka (2006) and Beneš et al. (2003).

\section{CONCLUSION}

We have shown that under a tail equivalence assumption the maximum domain of attraction of spheroid characteristics is preserved in their profiles up to a simple change of the respective parameter. These results can be used for the estimation of the distribution of spheroid characteristics extremes based on the observed profile characteristics extremes.

The exception is the class of prolate profiles with a bounded size. In this case it seems that the proposed analysis can only be used for the marginal distribution of the size or the shape factor. The study of the conditional distribution of the size given the shape factor and vice versa must probably be based on a different approach in this situation. 


\section{ACKNOWLEDGMENT}

The work is a part of the research project MSM 0021620839 financed by MSMT

The author thanks the referees for their very careful reviews and valuable comments.

\section{REFERENCES}

Beneš V, Bodlák K, Hlubinka D (2003). Stereology of extremes; FGM bivariate distributions. Method Comp Appl Prob 5:289-308.

Beirlant J, Goegebeur Y, Teugels J, Segers J, De Waal D, Ferro C (2004). Statistics of extremes. Theory and applications. Chichester: Wiley.

Chernozhukov V (2005). Extremal quantile regression. Ann Stat 33:806-39.

Coles S (2001). An introduction to statistical modeling of extreme values. London: Springer Verlag.

Cruz-Orive L-M (1976). Particle size-shape distributions; the general spheroid problem. J Microsc 107:235-53.

Drees H, Reiss R-D (1992). Tail behavior in Wicksell's corpuscle problem. In: Galambos J, Kátai J, eds. Probability Theory and Applications. Dordrecht: Kluwer, 205-20.

Embrechts P, Klüppelberg C, Mikosch T (1997). Modelling extremal events for insurance and finance. Berlin: Springer Verlag.

de Haan L (1970). On regular variation and its application to the weak convergence of sample extremes. Math
Centre Tracts 32. Amsterdam: Mathematisch Centrum Amsterdam.

Hlubinka D (2003a). Stereology of extremes; shape factor of spheroids. Extremes 5:5-24.

Hlubinka D (2003b). Stereology of extremes; size of spheroids. Math Bohem 128:419-38.

Hlubinka D (2006). Extremes of spheroid shape factor based on two dimensional profiles. Kybernetika 42:77-94.

Hlubinka D, Kotz S (2006) The generalized FGM distribution and its application to stereology of extremes. To appear.

Kötzer S, Molchanov I (2006). On the domain of attraction for the lower tail in Wicksell's corpuscle problem. In: Beneš V, Lechnerová R, Saxl I, eds. Proceedings $S^{4} G$. Praha: JČMF, 91-6.

Takahashi R (1987). Normalizing constants of a distribution which belongs to the domain of attraction of the Gumbel distribution. Stat Probabil Lett 5:197-200.

Takahashi R and Sibuya M (1996). The maximum size of the planar sections of random spheres and its application to metalurgy. Ann Inst Stat Math 48:127-44.

Takahashi R, Sibuya M (2002). Maximum size prediction in Wicksell's corpuscle problem for the exponential tail data. Extremes 5:55-70.

Wicksell SD (1925). The corpuscle problem I. Biometrika 17:84-99.

Wicksell SD (1926). The corpuscle problem II. Biometrika 18:152-72. 